Pathophysiology Haemostasis and Thrombosis

\title{
Mechanisms of Signal Transduction in Vascular Permeability: Potential Targets
}

\author{
Brian Eliceiri \\ La Jolla Institute for Molecular Medicine, San Diego, Calif., USA
}

Our research has focused on understanding the molecular basis of the requirement for receptors for the extracellular matrix (integrins) during growth factor-induced vascular responses, including angiogenesis and vascular permeability (VP). Significant progress has been made in the understanding of some of the fundamental biological differences between two angiogenic growth factors, vascular endothelial growth factor (VEGF) and basic fibroblast growth factor (bFGF). For example, we have previously shown that the ligation of specific integrins is required for a sustained intracellular signal (MAP kinase) during bFGF-induced angiogenesis (J Cell Biology 1998;140:1255-1263). As tumors are known to secrete combinations of growth factors such as bFGF and VEGF, it is imperative to understand how each one functions on its own to better understand the effects of combinations of these growth factors on angiogenesis and tumor growth in vivo. This strategy has led to the discovery of differences at the molecular level between these growth factor-induced cascades (Molecular Cell 1999;4:915-924). These results have translated not only into a better understanding of the signaling pathways following integrin ligation and growth factor receptor activation, but it has also uncovered a molecular cue which distinguishes between VEGF-induced blood vessel growth and blood vessel leakage/permeability. This work indicates that the VEGF-induced vascular growth can be distinguished from VEGF-induced VP in mutant mice that are deficient for the Src tyrosine kinase. These results have immediate

\begin{tabular}{ll}
\hline KARGER & ( ) 2003 S. Karger AG, Basel \\
Fax +4161306 1234 & 1424-8832/03/0337-0005\$19.50/0 \\
$\begin{array}{l}\text { E-Mail karger@karger.ch } \\
\text { www.karger.com }\end{array}$ & $\begin{array}{l}\text { Accessible online at: } \\
\text { www.karger.com/journals/pht }\end{array}$
\end{tabular}

consequences not only in cancer models in mice but also in mouse models of stroke. In murine stroke models, Src deficiency or blockade of Src activity provided cerebral protection following cerebral ischemia (Nature Med 2001;7: 222-227).

\section{The Role of Src in the VEGF-Induced Vascular Responses}

We have previously demonstrated that the signaling requirements for the VEGF pathway of angiogenesis were significantly different from that of the bFGF pathway of angiogenesis (Molecular Cell 1999;4:915-924). Specifically, a class of intracellular tyrosine kinases, known as the Src family kinases, was shown to be required for VEGF-induced but not bFGF-induced angiogenesis. This was demonstrated in both chick embryo and mouse models of angiogenesis. Inhibition of the Src family kinases was also sufficient to block human brain tumor cell growth in chick embryos. Furthermore, apoptosis was shown to be the mechanism responsible for the loss of vascular endothelium in VEGF-stimulated tissues following retroviral gene delivery of mutant Src genes. The use of retroviruses to deliver genes to block tumor growth and VEGF-induced angiogenesis was a form of 'gene therapy' for the chick and mouse models, used to dissect the molecular mechanism(s) between different growth factors. This work led to a further 
examination of the requirements of Src family kinases by studying mice deficient for each of the Src family kinases known to be expressed in vascular endothelium, namely Src, Fyn, and Yes.

Src-deficient mice injected in the dermis with an adenovirus expressing VEGF, underwent a markedly reduced level of vascular leakage/permeability compared to wildtype controls. This discovery led to an analysis of other tissues and other Src-related mutants such as Fyn- or Yes-deficient mice. Yes-deficient mice, like the Src-deficient mice, were also leakage resistant in response to the VEGF stimulus. In contrast, the Fyn-deficient mice appeared more like wild type controls, exhibiting normal robust vascular leakage in response to VEGF. The resistance to vascular leakage was also demonstrated in the brains of Src-deficient mice.

\section{Role of Src in Coordinating FAK/Integrin $\alpha v \beta 5$ Complexes}

Progress is being made in the understanding of the intracellular signaling events that follow VEGF-induced angiogenesis leading to the activation of the Src tyrosine kinase and downstream events. While integrin ligation and growth factor receptor stimulation leads to Src activation, focal adhesion kinase (FAK) is also activated. FAK is known to form a complex with Src that leads to the activation and phosphorylation of Src and further phosphorylation by Src of several putative tyrosine sites in FAK. Therefore, mutant constructs of FAK were expressed by retroviral gene delivery in the chick embryo. Both bFGF- and VEGF-induced angiogenesis were blocked by a mutant FAK gene. To further study the biochemical associations of FAK in the VEGF pathway of angiogenesis, immunoprecipitations of the integrins $\alpha \mathrm{v} \beta 5$ or $\alpha \mathrm{v} \beta 3$ were performed from VEGFstimulated or quiescent endothelial cells. VEGF induced the formation of a FAK/ $\alpha v \beta 5$ complex, whereas the FAK was constitutively associated with the $\alpha \mathrm{v} \beta 3$. This has led to further experiments in which mutant Src or mutant FAK has been shown to block the VEGF-induced formation of a FAK- $\alpha v \beta 5$ complex. Furthermore, an active Src cDNA can induce angiogenesis, which is selectively blocked by $\alpha v \beta 5$, and is sufficient to induce a FAK- $\alpha v \beta 5$ complex. Phosphorylation-state specific antibodies directed against each of the known important tyrosines in FAK and Src are being used to further map which sites are activated in the endogenous FAK or Src upon VEGF stimulation of blood vessels. This work has been published in The Journal of Cell Biology 2002;157:149-159.

\section{Progress on the Identification of the Role of Src in Tumor Growth/Metastasis and Stroke}

The analysis of the physiological role of VP in Srcdeficient mice is being addressed in mouse models of tumor growth/metastasis and stroke. For the tumor studies, syngeneic tumors of the same mouse strain are being introduced into the wildtype or Src-deficient mice. Data from melanoma tumors indicates that tumors grow significantly larger in Src deficient mice, which have leakage resistant vessels, compared to wildtype controls. This surprising finding suggests that during tumor growth, a normal leakageresponsive blood vessel, which grow smaller tumors, is better for an animal than a Src-deficient blood vessel, which can grow very large tumors. This work is being extended in syngeneic and transgenic models of spontaneous metastasis. These studies are designed to address the role of Srcmediated VP on the deposition of matrix proteins into the perivascular space (i.e. fibrin) and the effect on the metastasis of tumor cells in vivo.

A second important vascular disease is stroke in which stroke-induced edema leading to brain damage. In mouse models of stroke induced by ligation of the middle cerebral artery or clotting, Src-deficient mice are protected from brain damage (Nature Med 2001;7:222-227). Furthermore, a synthetic inhibitor of Src kinase also blocks brain damage in a wildtype mouse with a stroke. The reduced infarct volume in either Src-deficient mice or Src inhibitor-treated mice following cerebral ischemia, suggests a potent clinical value from antagonizing the Src pathway to reduce vascular leakage and permeability. 